\title{
Human Drug Compounding Outsourcing Facility
}

National Cancer Institute

\section{Source}

National Cancer Institute. Human Drug Compounding Outsourcing Facility. NCI

Thesaurus. Code C112113.

A site designated as an establishment for outsourcing of human drug compounding. 